\title{
Commentary to paper "Feasibility of laparoscopic cholecystectomy for acute cholecystitis beyond 72 hours of symptom onset"
}

\author{
Dario Borreca $^{1}$ (D) Andrea Borasi ${ }^{1} \cdot$ Paolo De Paolis $^{1}$
}

Received: 10 November 2016/Accepted: 11 November 2016/Published online: 16 November 2016

(C) Italian Society of Surgery (SIC) 2016

Cholelithiasis is a daily diagnosed pathology in clinical practice, all over the world. Gallstone disease is prevalent in $10 \%$ of the population, and up to $23 \%$ can develop into acute cholecystitis [1]. Laparoscopic cholecystectomy (LC) represents nowadays the "gold standard" for cholecystolithiasis since the late 1990s, but the best timing to treat a challenging concomitant condition, such as an acute cholecystitis, is still debated. As operating acutely was previously believed to be more technically challenging due to distorted anatomy from acute inflammation, a cooling off period has been advocated, employing a conservative treatment with empiric antibiotic therapy followed by elective surgery several weeks later. Conversely, this approach could require further readmissions for recurrent episodes; furthermore, chronic inflammation, leading to fibrosis and adhesions, may make for a difficult dissection in delayed LC. Following this background, several authors advocate an early surgery, within first $72 \mathrm{~h}$ from the onset of the symptoms, whereas others assume acceptable to wait even 4-6 weeks or more [1]. The debated key issues reside in intraoperative complications, needing a laparotomic conversion, operative time, cost containment, and reduction of length of stay (LOS), evaluating the subsequent quality of life, and improving the safety of the surgical act.

A recent review by Gurusamy et al. [1] has shown as the proportion of people who developed a bile duct injury that was significantly lower in the early group, with no

Dario Borreca

d.borreca@policlinicoumberto1.it;

dario.borreca@gradenigo.it

1 Department of General Surgery, Humanitas Gradenigo Research Hospital, Corso Regina Margherita 8/10, 10153 Turin, Italy differences in other serious adverse events. However, the conversion rate was not significantly different. Similarly, also the operating time was comparable. Interestingly, Onoe et al. [2] have identified at univariate analysis C-reactive protein as predictive variable for laparotomic conversion; high CRP values ( $>10 \mathrm{mg} / \mathrm{dl}$ ) have been related to the failure to create a critical view of safety, leading to a more accurate surgical strategy choice, in relation to the individual case.

Pursuing the topic, a comprehensive meta-analysis by Cao et al. [3] demonstrates that these benefits will still present, although on a smaller scale, in the subgroup operated after $72 \mathrm{~h}$, but within 7 days. This suggests that the " $72 \mathrm{~h}$ rule" is an optimal timing; however, patients operated after this window still benefit from an early LC, rather than a non-operative management. This finding is particularly relevant because of the known logistical issues to schedule the procedures, lacking an appropriate access in our operating theaters.

Two further disadvantages of delayed approach are the risk of unsuccessful conservative management and the chance of a recurrent episode. According to up-to-date literature, first trouble accounts up to $16 \%$, requiring an urgent LC, while an unnecessary delay results in a $23 \%$ recurrent rate, which can lead to an increased morbidity and mortality, increasing costs and need for facilities [4]. Total hospital stay was found to be shorter by 4 days in early treated patients. Each day of saved hospitalization was quantified in 300€; decreased LOS could reflect a sensible reduction of costs, but it needs to be formally verified [5]. Furthermore, despite the small number of studies, a formal economical analysis revealed that a policy of early intervention results in an overall better quality of life for people at 1-year post-presentation, with an early 
resume by 11 days of working activity, and lower indirect costs.

Consequently, it does not seem to be justified to wait more than $72 \mathrm{~h}$ to perform LC in acute cholecystitis; it is, moreover, assertible that in agreement with the paper of Onoe et al. [3], an early treatment in suitable patients leads to higher success rate, less adverse events, with shorter hospital stay, and less costs, but with technical and organization efforts. Despite the current evidences, surgical practice varies significantly worldwide, with a range of $11-89 \%$ of surgeons performing an early approach; furthermore, it is still maintained the culture that acute cholecystitis is not a surgical emergency [3]. To change the attitude of this challenging pathology, the surgeons should become aware of clearly benefits demonstrated in the commented study and new models need to be developed to improve the management of emergency surgical cases, such as dedicated acute surgical units, overcoming aforementioned drawbacks.

\section{Compliance with ethical standards}

Conflict of interest Drs. Dario Borreca, Andrea Borasi, and Paolo De Paolis have no conflicts of interests or financial ties to disclose.

Ethical standard All procedures performed in studies involving human participants were in accordance with the ethical standards of the institutional and/or national research committee and with the 1964 Helsinki declaration and its later amendments or comparable ethical standards.
Research involving human participants and/or animals This article does not contain any studies with human participants or animals performed by any of the authors.

Informed consent For this type of study formal consent is not required.

\section{References}

1. Gurusamy KS, Davidson C, Gluud C, Davidson BR (2013) Early versus delayed laparoscopic cholecystectomy for people with acute cholecystitis. Cochrane Database Syst Rev 6:CD005440. doi:10. 1002/14651858.CD005440.pub3

2. Onoe S, Kaneoka Y, Maeda A, Takayama Y, Fukami Y (2016) Feasibility of laparoscopic cholecystectomy for acute cholecystitis beyond 72 hours of symptom onset. Updates Surg. doi:10.1007/ s13304-016-0409-5

3. Cao AM, Eslick GD, Cox MR (2016) Early laparoscopic cholecystectomy is superior to delayed acute cholecystitis: a meta-analysis of case-control studies. Surg Endosc 30:1172-1182. doi:10.1007/s00464-015-4325-4

4. Gutt CN, Encke J, Köninger J, Harnoss JC, Weigand K, Kipfmüller K, Schunter O, Götze T, Golling MT, Menges M, Klar E, Feilhauer K, Zoller WG, Ridwelski K, Ackmann S, Baron A, Schön MR, Seitz HK, Daniel D, Stremmel W, Büchler MW (2013) Acute cholecystitis: early versus delayed cholecystectomy, a multicenter randomized trial (ACDC study, NCT00447304). Ann Surg 258(3):385-393. doi:10.1097/SLA.0b013e3182a1599b

5. Borreca D, Bona A, Bellomo MP, Borasi A, De Paolis P (2015) 'Ultra-rapid' sequential treatment in cholecystocholedocholithiasis: alternative same-day approach to laparoendoscopic rendezvous. Updates Surg. 67(4):449-454. doi:10.1007/s13304-0150339-7 